\title{
Multi-dimensional constraint relativistic mean field model and applications in actinide and transfermium nuclei
}

\author{
Bing-Nan $\mathrm{Lu}^{1}$, Jie Zhao ${ }^{1}$, En-Guang Zhao ${ }^{1,2}$ and Shan-Gui Zhou ${ }^{1,2 *}$ \\ ${ }^{1}$ State Key Laboratory of Theoretical Physics, Institute of Theoretical Physics, \\ Chinese Academy of Sciences, Beijing 100190, China \\ ${ }^{2}$ Center of Theoretical Nuclear Physics, National Laboratory of Heavy Ion Accelerator, \\ Lanzhou 730000, China
}

PACS ReF: 21.60.Jz, 24.75.+i, 25.85.-w, 27.90.+b

Nov 14, 2013

\begin{abstract}
In this contribution we present some results of potential energy surfaces of actinide and transfermium nuclei from multi-dimensional constrained relativistic mean field (MDC-RMF) models. Recently we developed multi-dimensional constrained covariant density functional theories (MDC-CDFT) in which all shape degrees of freedom $\beta_{\lambda \mu}$ with even $\mu$ are allowed and the functional can be one of the following four forms: the meson exchange or point-coupling nucleon interactions combined with the non-linear or density-dependent couplings. In MDC-RMF models, the pairing correlations are treated with the BCS method. With MDC-RMF models, the potential energy surfaces of even-even actinide nuclei were investigated and the effect of triaxiality on the fission barriers in these nuclei was discussed. The non-axial reflection-asymmetric $\beta_{32}$ shape in some transfermium nuclei with $N=150$, namely ${ }^{246} \mathrm{Cm},{ }^{248} \mathrm{Cf},{ }^{250} \mathrm{Fm}$, and ${ }^{252}$ No were also studied.
\end{abstract}

\section{Introduction}

The occurrence of spontaneous symmetry breaking in atomic nuclei leads to various nuclear shapes which can usually be described by the parametrization of the nuclear surface or the nucleon density distribution $[1,2]$. In mean-field calculations, the following parametrization

$$
\beta_{\lambda \mu}=\frac{4 \pi}{3 A R^{\lambda}}\left\langle Q_{\lambda \mu}\right\rangle
$$

is usually used, where $Q_{\lambda \mu}$ are the mass multipole operators. In Fig. 1, a schematic show is given for some typical nuclear shapes. The majority of observed nuclear shapes is of spheroidal form which is usually described by $\beta_{20}$. Higher-order deformations with $\lambda>2$ such as $\beta_{30}$ also appear in certain atomic mass regions [3]. In addition, non-axial shapes in atomic nuclei, in particular, the nonaxial-quadrupole (triaxial) deformation $\beta_{22}$ has been studied both experimentally and theoretically [4-6]. The influence of the nonaxial octupole $\beta_{32}$ deformation on the low-lying spectra has been also investigated [7-16].

\footnotetext{
*e-mail:sgzhou@itp.ac.cn
}

\begin{tabular}{|c|c|c|c|}
\hline$\beta_{\lambda \mu}=0$ & $\beta_{20}>0$ & $\beta_{20}<0$ & $\beta_{40}>0$ \\
\hline & 0 & \\
\hline$\beta_{22} \neq 0$ & $\beta_{30} \neq 0$ & & \\
\hline & & \\
\hline
\end{tabular}

Fig. 1: (Color online) A schematic show of some typical nuclear shapes. From left to right, the 1st row: (a) Sphere, (b) Prolate spheroid, (c) Oblate spheroid, (d) Hexadecapole shape, and the second row: (e) Triaxial ellipsoid, (f) Reflection symmetric octupole shape, (g) Tetrahedron, (h) Reflection asymmetric octupole shape with very large quadrupole deformation and large hexadecapole deformation. Taken from Ref. [17].

In nuclear fission study, various shape degrees of freedom play important and different roles in the occurrence and in determining the heights of the inner and outer barriers in actinide nuclei (in these nuclei double-humped fission barriers usually appear). For example, the inner fission barrier is usually lowered when the triaxial deformation is allowed, while for the outer barrier the reflection asymmetric (RA) shape is favored [18-22,24, 25].

In order to give a microscopic and self-consistent description of the potential energy surface (PES) with more shape degrees of freedom included, multi-dimensional constrained covariant density functional theories were developed recently $[26,27]$. In these theories, all shape degrees of freedom $\beta_{\lambda \mu}$ with even $\mu$ are allowed. In this contribution, we present two recent applications of these theories: the PES's of actinide nuclei and the non-axial reflectionasymmetric $\beta_{32}$ shape in some transfermium nuclei. In Section 2, the formalism of our multi-dimensional constrained covariant density functional theories will be given 
briefly. The results and discussions are presented in Section 3. Finally we give a summary in Section 4 .

\section{Formalism}

The details of the formalism for covariant density functional theories can be found in Refs. [28-33]. The CDFT functional in our multi-dimensional constrained calculations can be one of the following four forms: the meson exchange or point-coupling nucleon interactions combined with the non-linear or density-dependent couplings [26, $27,34,35]$. Here we show briefly the one corresponding to the non-linear point coupling (NL-PC) interactions. The starting point of the relativistic NL-PC density functional is the following Lagrangian:

$$
\mathcal{L}=\bar{\psi}\left(i \gamma_{\mu} \partial^{\mu}-M\right) \psi-\mathcal{L}_{\text {lin }}-\mathcal{L}_{\text {nl }}-\mathcal{L}_{\text {der }}-\mathcal{L}_{\text {Cou }},
$$

where

$$
\begin{aligned}
\mathcal{L}_{\text {lin }}= & \frac{1}{2} \alpha_{S} \rho_{S}^{2}+\frac{1}{2} \alpha_{V} \rho_{V}^{2}+\frac{1}{2} \alpha_{T S} \vec{\rho}_{T S}^{2}+\frac{1}{2} \alpha_{T V} \vec{\rho}_{T V}^{2} \\
\mathcal{L}_{\mathrm{nl}}= & \frac{1}{3} \beta_{S} \rho_{S}^{3}+\frac{1}{4} \gamma_{S} \rho_{S}^{4}+\frac{1}{4} \gamma_{V}\left[\rho_{V}^{2}\right]^{2} \\
\mathcal{L}_{\text {der }}= & \frac{1}{2} \delta_{S}\left[\partial_{\nu} \rho_{S}\right]^{2}+\frac{1}{2} \delta_{V}\left[\partial_{\nu} \rho_{V}\right]^{2}+\frac{1}{2} \delta_{T S}\left[\partial_{\nu} \vec{\rho}_{T S}\right]^{2} \\
& +\frac{1}{2} \delta_{T V}\left[\partial_{\nu} \vec{\rho}_{T V}\right]^{2} \\
\mathcal{L}_{\text {Cou }}= & \frac{1}{4} F^{\mu \nu} F_{\mu \nu}+e \frac{1-\tau_{3}}{2} A_{0} \rho_{V}
\end{aligned}
$$

are the linear coupling, nonlinear coupling, derivative coupling, and the Coulomb part, respectively. $M$ is the nucleon mass, $\alpha_{S}, \alpha_{V}, \alpha_{T S}, \alpha_{T V}, \beta_{S}, \gamma_{S}, \gamma_{V}, \delta_{S}, \delta_{V}, \delta_{T S}$, and $\delta_{T V}$ are coupling constants for different channels and $e$ is the electric charge. $\rho_{S}, \vec{\rho}_{T S}, \rho_{V}$, and $\vec{\rho}_{T V}$ are the isoscalar density, isovector density, time-like components of isoscalar current, and time-like components of isovector current, respectively. The densities and currents are defined as

$$
\begin{aligned}
\rho_{S}=\bar{\psi} \psi, & \vec{\rho}_{T S}=\bar{\psi} \vec{\tau} \psi, \\
\rho_{V}=\bar{\psi} \gamma^{0} \psi, & \vec{\rho}_{T V}=\bar{\psi} \vec{\tau} \gamma^{0} \psi .
\end{aligned}
$$

Starting from the above Lagrangian, using the Slater determinants as trial wave functions and neglecting the Fock term as well as the contributions to the densities and currents from the negative energy levels, one can derive the equations of motion for the nucleons,

$$
\hat{h} \psi_{i}=(\boldsymbol{\alpha} \cdot \vec{p}+\beta(M+S(\vec{r}))+V(\vec{r})) \psi_{i}=\epsilon_{i} \psi_{i},
$$

where the potentials $V(\boldsymbol{r})$ and $S(\boldsymbol{r})$ are calculated as

$$
\begin{aligned}
S= & \alpha_{S} \rho_{S}+\beta_{S} \rho_{S}^{2}+\gamma_{S} \rho_{S}^{3}+\delta_{S} \triangle \rho_{S} \\
& +\left(\alpha_{T S} \rho_{T S}+\delta_{T S} \triangle \rho_{T S}\right) \tau_{3}, \\
V= & \alpha_{V} \rho_{V}+\gamma_{V} \rho_{V}^{3}+\delta_{V} \triangle \rho_{V} W \\
& +\left(\alpha_{T V} \rho_{T V}+\delta_{T V} \triangle \rho_{T V}\right) \tau_{3} .
\end{aligned}
$$

An axially deformed harmonic oscillator (ADHO) basis is adopted for solving the Dirac equation [26, 27, 36]. The ADHO basis is defined as the eigen solutions of the Schrodinger equation with an ADHO potential $[37,38]$,

$$
\left[-\frac{\hbar^{2}}{2 M} \nabla^{2}+V_{B}(z, \rho)\right] \Phi_{\alpha}(\boldsymbol{r} \sigma)=E_{\alpha} \Phi_{\alpha}(\boldsymbol{r} \sigma),
$$

where

$$
V_{B}(z, \rho)=\frac{1}{2} M\left(\omega_{\rho}^{2} \rho^{2}+\omega_{z}^{2} z^{2}\right),
$$

is the axially deformed $\mathrm{HO}$ potential and $\omega_{z}$ and $\omega_{\rho}$ are the oscillator frequencies along and perpendicular to $z$ axis, respectively. These basis states are also eigen functions of the $z$ component of the angular momentum $j_{z}$ with eigen values $K=m_{l}+m_{s}$. For any basis state $\Phi_{\alpha}(\boldsymbol{r} \sigma)$, the time reversal state is defined as $\Phi_{\bar{\alpha}}(\boldsymbol{r} \sigma)=\mathcal{T} \Phi_{\alpha}(\boldsymbol{r} \sigma)$, where $\mathcal{T}=i \sigma_{y} K$ is the time reversal operator and $K$ is the complex conjugation. Apparently we have $K_{\bar{\alpha}}=-K_{\alpha}$ and $\pi_{\bar{\alpha}}=\pi_{\alpha}$. These basis states form a complete set for expanding any two-component spinors. For a Dirac spinor with four components,

$$
\psi_{i}(\boldsymbol{r} \sigma)=\left(\begin{array}{c}
\sum_{\alpha} f_{i}^{\alpha} \Phi_{\alpha}(\boldsymbol{r} \sigma) \\
\sum_{\alpha}^{\alpha} g_{i}^{\alpha} \Phi_{\alpha}(\boldsymbol{r} \sigma)
\end{array}\right),
$$

where the sum runs over all the possible combination of the quantum numbers $\alpha=\left\{n_{z}, n_{r}, m_{l}, m_{s}\right\}$, and $f_{i}^{\alpha}$ and $g_{i}^{\alpha}$ are the expansion coefficients. In practical calculations, one should truncate the basis in a proper way $[26,27,36]$.

The nucleus is assumed to be symmetric under the $V_{4}$ group, that is, all the potentials and densities can be expanded as

$$
f(\rho, \varphi, z)=f_{0}(\rho, z) \frac{1}{\sqrt{2 \pi}}+\sum_{n=1}^{\infty} f_{n}(\rho, z) \frac{1}{\sqrt{\pi}} \cos (2 n \varphi),
$$

The PES is obtained by the constrained self-consistent calculation,

$$
E^{\prime}=E_{\mathrm{RMF}}+\sum_{\lambda \mu} \frac{1}{2} C_{\lambda \mu} Q_{\lambda \mu}
$$

where the variables $C_{\lambda \mu}$ 's change their values during the iteration.

Both the BCS approach and the Bogoliubov transformation are implemented in our model to take into account the pairing effects. For convenience, we name the MDC-CDFT with the BCS approach for the pairing as the MDC-RMF model and that with the Bogoliubov transformation as the MDC-RHB model. More details of the multi-dimensional constraint covariant density functional theories can be found in Refs. [26,27].

\section{Results and discussions}

\subsection{PES's of actinides}

In Refs. [26,27], one- (1-d), two- $(2-\mathrm{d})$, and threedimensional (3-d) constrained calculations were performed 


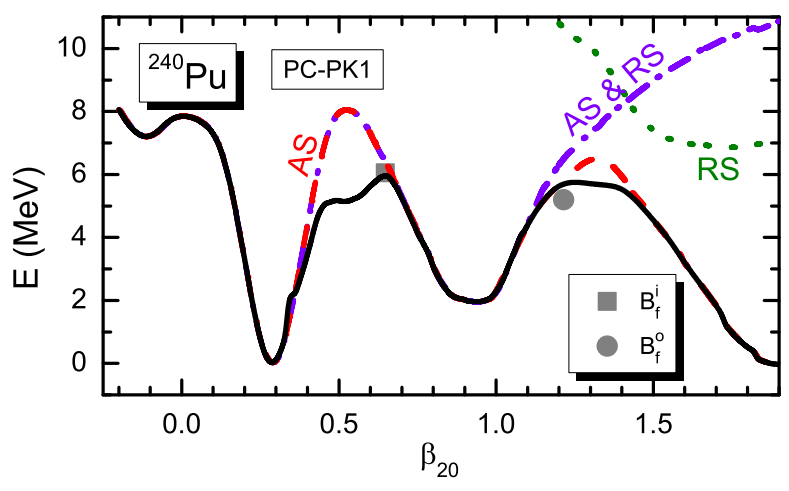

Fig. 2: (Color online) Potential energy curves of ${ }^{240} \mathrm{Pu}$ with various self-consistent symmetries imposed. The solid black curve represents the calculated fission path with $V_{4}$ symmetry imposed: the red dashed curve is that with axial symmetry (AS) imposed, the green dotted curve that with reflection symmetry (RS) imposed, the violet dot-dashed line that with both symmetries (AS \& RS) imposed. The empirical inner (outer) barrier height $B_{\text {emp }}$ is denoted by the grey square (circle). The energy is normalized with respect to the binding energy of the ground state. The parameter set used is PC-PK1. Taken from Ref. [26].

for the actinide nucleus ${ }^{240} \mathrm{Pu}$. The MDC-RMF model with the parameter set PC-PK1 [39] was used. In Fig. 2 we show the 1-d potential energy curves from an oblate shape with $\beta_{20}$ about -0.2 to the fission configuration with $\beta_{20}$ beyond 2.0 which are obtained from calculations with different self-consistent symmetries imposed: the axial (AS) or triaxial (TS) symmetries combined with reflection symmetric (RS) or asymmetric cases. The importance of the triaxial deformation on the inner barrier and that of the octupole deformation on the outer barrier are clearly seen: The triaxial deformation reduces the inner barrier height by more than $2 \mathrm{MeV}$ and results in a better agreement with the empirical value [22]; the RA shape is favored beyond the fission isomer and lowers very much the outer fission barrier [21]. Besides these features, it was found for the first time that the outer barrier is also considerably lowered by about $1 \mathrm{MeV}$ when the triaxial deformation is allowed. In addition, a better reproduction of the empirical barrier height can be seen for the outer barrier. It has been stressed that this feature can only be found when the axial and reflection symmetries are simultaneously broken [26].

Two-dimensional PES's in the $\left(\beta_{20}, \beta_{22}\right)$ plane near the inner and outer barriers are shown in Figs. 3 and 4 , respectively. Starting from the axially symmetric ground state, the nucleus goes through the triaxial valley to the isometric state. The inner barrier is located at $\beta_{20} \approx 0.65$ and $\beta_{22} \approx 0.06$. The isomeric state keeps an axially symmetric shape. As $\beta_{20}$ further increases, the nucleus goes through a triaxial valley again, and then goes fission. The outer barrier is located at $\beta_{20} \approx 1.21, \beta_{22} \approx 0.02$, and $\beta_{30} \approx 0.37$.

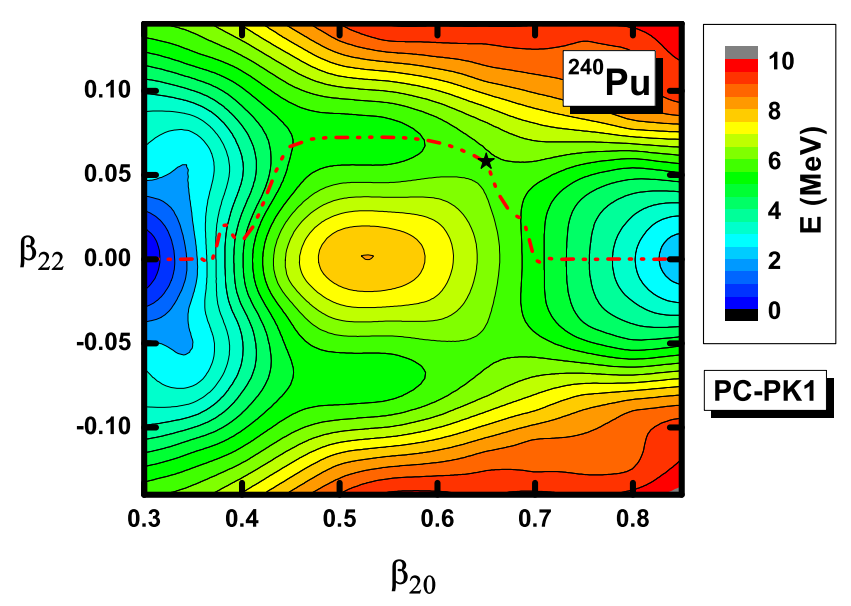

Fig. 3: (Color online) Potential energy surfaces of ${ }^{240} \mathrm{Pu}$ in the $\left(\beta_{20}, \beta_{22}\right)$ plane around the inner barrier. The energy is normalized with respect to the binding energy of the ground state. The least-energy fission path is represented by a dashdotted line. The saddle point is denoted by the full star. The contour interval is $0.5 \mathrm{MeV}$.

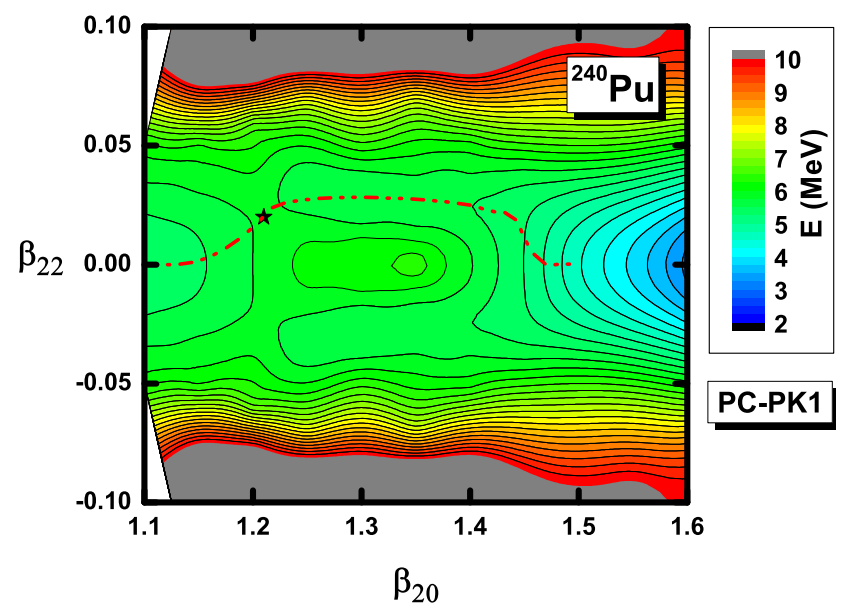

Fig. 4: (Color online) Potential energy surfaces of ${ }^{240} \mathrm{Pu}$ in the $\left(\beta_{20}, \beta_{22}\right)$ plane around the outer barrier. The energy is normalized with respect to the binding energy of the ground state. The least-energy fission path is represented by a dashdotted line. The saddle point is denoted by the full star. The contour interval is $0.25 \mathrm{MeV}$. 


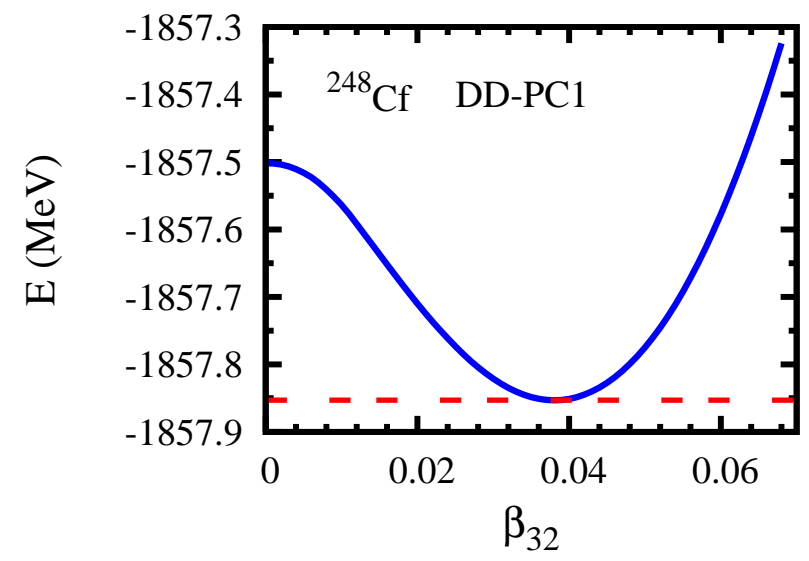

Fig. 5: (Color online) The binding energy $E$ (relative to the ground state) for ${ }^{248} \mathrm{Cf}$ as a function of the non-axial octupole deformation parameter $\beta_{32}$.

A systematic study of even-even actinide nuclei has been carried out and the results were presented in Ref. [27] where we have shown that the triaxial deformation lowers the outer barriers of these actinide nuclei by about $0.5 \sim 1$ $\mathrm{MeV}$ (about $10 \sim 20 \%$ of the barrier height).

\section{2. $Y_{32}$-correlations in $N=150$ isotones}

It has been anticipated that the tetrahedral shape $\left(\beta_{\lambda \mu}=\right.$ 0 , if $\lambda \neq 3$ and $\mu \neq 2$ ) may appear in the ground states of some nuclei with special combinations of the neutron and proton numbers $[9,12,16]$. The tetrahedral symmetrydriven quantum effects may also lead to a large increase of binding energy in superheavy nuclei [40]. However, no solid experimental evidence has been found for nuclei with tetrahedral shapes. On the other hand, $\beta_{32}$ deformation may appear together with other shape degrees of freedome, say, $\beta_{2}$. For example, it has been proposed that the non-axial octupole $Y_{32}$-correlation results in the experimentally observed low-energy $2^{-}$bands in the $N=150$ isotones [41] and the RASM calculations reproduces well the experimental observables of these $2^{-}$bands [42].

In Ref. [43] the non-axial reflection-asymmetric $\beta_{32}$ deformation in $N=150$ isotones, namely ${ }^{246} \mathrm{Cm},{ }^{248} \mathrm{Cf}$, ${ }^{250} \mathrm{Fm}$, and ${ }^{252} \mathrm{No}$ was investigated using the MDC-RMF model with the parameter set DD-PC1 [44]. It was found that due to the interaction between a pair of neutron orbitals, [734]9/2 originating from $\nu j_{15 / 2}$ and [622]5/2 originating from $\nu g_{9 / 2}$, and that of a pair of proton orbitals, [521]3/2 originating from $\pi f_{7 / 2}$ and [633]7/2 originating from $\pi i_{13 / 2}$, rather strong non-axial octupole $Y_{32}$ effects appear in ${ }^{248} \mathrm{Cf}$ and ${ }^{250} \mathrm{Fm}$ which are both well deformed with large axial-quadrupole deformations, $\beta_{20} \approx 0.3$.

In Fig. 5 , the potential energy curve, i.e., the total binding energy as a function of $\beta_{32}$ was shown for ${ }^{248} \mathrm{Cf}$. At each point of the potential energy curve, the energy is minimized automatically with respect to other shape degrees of freedom such as $\beta_{20}, \beta_{22}, \beta_{30}$, and $\beta_{40}$, etc. One finds in this curve a clear pocket with the depth more than 0.3 $\mathrm{MeV}$. Similar potential energy curve was also obtained for ${ }^{250} \mathrm{Fm}$. For ${ }^{246} \mathrm{Cm}$ and ${ }^{252} \mathrm{No}$, only a shallow minimum develops along the $\beta_{32}$ shape degree of freedom. It was also shown that the evolution of the non-axial octupole $\beta_{32}$ effect along the $N=150$ isotonic chain is not very sensitive to the form of the energy density functional and the parameter set we used [43].

Both the non-axial octupole parameter $\beta_{32}$ and the energy gain due to the $\beta_{32}$-distortion reach maximal values at ${ }^{248} \mathrm{Cf}$ in the four nuclei along the $N=150$ isotonic chain [43]. This is consistent with the analysis given in Refs. $[42,45]$ and the experimental observation that in ${ }^{248} \mathrm{Cf}$, the $2^{-}$state is the lowest among these nuclei [41]. These results indicate a strong $Y_{32}$-correlation in these nuclei.

\section{Summary}

In this contribution we present the formalism and some applications of the multi-dimensional constrained relativistic mean field (MDC-RMF) models in which all shape degrees of freedom $\beta_{\lambda \mu}$ with even $\mu$ are allowed. The potential energy surfaces (curves) of actinide nuclei and the effect of the triaxiality on the first and second fission barriers were investigated. It is found that besides the octupole deformation, the triaxiality also plays an important role upon the second fission barriers. The non-axial reflectionasymmetric $\beta_{32}$ shape in $N=150$ isotones were studied and rather strong non-axial octupole $Y_{32}$ effects have been found in ${ }^{248} \mathrm{Cf}$ and ${ }^{250} \mathrm{Fm}$ which are both well deformed with large axial-quadrupole deformations, $\beta_{20} \approx 0.3$.

\section{Acknowledgement}

This work has been supported by Major State Basic Research Development Program of China (Grant No. 2013CB834400), National Natural Science Foundation of China (Grant Nos. 11121403, 11175252, 11120101005, 11211120152, and 11275248), the Knowledge Innovation Project of Chinese Academy of Sciences (Grant No. KJCX2-EW-N01). The results described in this paper are obtained on the ScGrid of Supercomputing Center, Computer Network Information Center of Chinese Academy of Sciences.

\section{References}

[1] Bohr A and Mottelson B R 1998 Nuclear Structure vol I (World Scientific)

[2] Ring P and Schuck P 1980 The Nuclear Many-Body Problem (Springer-Verlag Berlin, Heidelberg, and New York)

[3] Butler P A and Nazarewicz W 1996 Rev. Mod. Phys. 68 349-421 
[4] Starosta K, et al. 2001 Phys. Rev. Lett. 86 971-974

[5] Odegard S W, et al. 2001 Phys. Rev. Lett. 865866 5869

[6] Meng J and Zhang S Q 2010 J. Phys. G: Nucl. Phys. 37 064025-12

[7] Hamamoto I, Mottelson B, Xie H and Zhang X Z 1991 Z. Phys. D 21 163-175

[8] Skalski J 1991 Phys. Rev. C 43 140-145

[9] Li X and Dudek J 1994 Phys. Rev. C 49 R1250$\mathrm{R} 1252$

[10] Takami S, Yabana K and Matsuo M 1998 Phys. Lett. B $431242-248$

[11] Yamagami M, Matsuyanagi K and Matsuo M 2001 Nucl. Phys. A 693 579-602

[12] Dudek J, Gozdz A, Schunck N and Miskiewicz M 2002 Phys. Rev. Lett. 88 252502-4

[13] Dudek J, Curien D, Dubray N, Dobaczewski J, Pangon V, Olbratowski P and Schunck N 2006 Phys. Rev. Lett. 97 072501-4

[14] Olbratowski P, Dobaczewski J, Powalowski P, Sadziak M and Zberecki K 2006 Int. J. Mod. Phys. E $15333-338$

[15] Zberecki K, Magierski P, Heenen P H and Schunck N 2006 Phys. Rev. C $\mathbf{7 4} 051302(\mathrm{R})-5$

[16] Dudek J, Gozdz A, Mazurek K and Molique H 2010 J. Phys. G: Nucl. Phys. 37 064032-16

[17] Lu B N 2012 Multi-dimensional constrained relativistic mean field theory and the potential energy surfaces and fission barriers of actinide nuclei Ph.D. thesis Institute of Theoretical Physics, Chinese Academy of Sciences in Chinese

[18] Pashkevich V V 1969 Nucl. Phys. A 133 400-404

[19] Möller P and Nilsson S G 1970 Phys. Lett. B 31283 286

[20] Girod M and Grammaticos B 1983 Phys. Rev. C 27 $2317-2339$

[21] Rutz K, Maruhn J A, Reinhard P G and Greiner W 1995 Nucl. Phys. A 590 680-702

[22] Abusara H, Afanasjev A V and Ring P 2010 Phys. Rev. C 82 044303-11

[23] Abusara H, Afanasjev A V and Ring P 2012 Phys. Rev. C 85 024314-17

[24] Prassa V, Nikšić T, Lalazissis G A and Vretenar D 2012 Phys. Rev. C 86 024317-12
[25] Prassa V, Niksic T and Vretenar D 2013 Phys. Rev. C 88 044324-11

[26] Lu B N, Zhao E G and Zhou S G 2012 Phys. Rev. C 85 011301(R)-5 (Preprint 1110.6769)

[27] Lu B N, Zhao J, Zhao E G and Zhou S G 2013 arXiv:1304.2513 [nucl-th]

[28] Serot B D and Walecka J D 1986 Adv. Nucl. Phys. $161-327$

[29] Reinhard P G 1989 Rep. Prog. Phys. 52 439-514

[30] Ring P 1996 Prog. Part. Nucl. Phys. 37 193-263

[31] Vretenar D, Afanasjev A, Lalazissis G and Ring P 2005 Phys. Rep. 409 101-259

[32] Meng J, Toki H, Zhou S G, Zhang S Q, Long W H and Geng L S 2006 Prog. Part. Nucl. Phys. 57 470-563 (Preprint nucl-th/0508020)

[33] Nikšić T, Vretenar D and Ring P 2011 Prog. Part. Nucl. Phys. 66 519-548

[34] Lu B N, Zhao J, Zhao E G and Zhou S G 2012 EPJ Web Conf. 3805003 (Preprint 1303.0621)

[35] Lu B N, Zhao J, Zhao E G and Zhou S G 2013 arXiv:1304.6830 [nucl-th] Proceedings of the XXXVI Mexican Symposium on Nuclear Physics, Cocoyoc, Morelos, Mexico, Jan. 7-10, 2013

[36] Lu B N, Zhao E G and Zhou S G 2011 Phys. Rev. C 84 014328-10 (Preprint 1104.4638)

[37] Gambhir Y K, Ring P and Thimet A 1990 Ann. Phys. $198132-179$

[38] Ring P, Gambhir Y K and Lalazissis G A 1997 Comput. Phys. Commun. 105 77-97

[39] Zhao P W, Li Z P, Yao J M and Meng J 2010 Phys. Rev. C 82 054319-14

[40] Chen Y and Gao Z 3013 Nucl. Phys. Rev. 30 278-283

[41] Robinson A P, et al. 2008 Phys. Rev. C 78 034308-6

[42] Chen Y S, Sun Y and Gao Z C 2008 Phys. Rev. C 77 061305(R)-5

[43] Zhao J, Lu B N, Zhao E G and Zhou S G 2012 Phys. Rev. C 86 057304-4 (Preprint 1209.6567)

[44] Nikšić T, Vretenar D and Ring P 2008 Phys. Rev. C $78034318-19$

[45] Jolos R V, Malov L A, Shirikova N Y and Sushkov A V 2011 J. Phys. G: Nucl. Part. Phys. 38 115103- 